\title{
Integrated GPS/TOA Navigation using a Positioning and Communication Software Defined Radio
}

\author{
Alison Brown and Janet Nordlie \\ NAVSYS Corporation \\ 14960 Woodcarver Road \\ Colorado Springs, CO 80921
}

\begin{abstract}
While GPS is the natural choice for providing navigation in an outdoors environment, the urban environment places a significant challenge for positioning using GPS. The GPS signals can be significantly attenuated, and often completely blocked, inside buildings or in urban canyons. In this paper a networked radionavigation approach is described that augments the GPS signals with Time of Arrival (TOA) observations using a Software Defined Radio (SDR).

The Software Defined Radio developed by NAVSYS includes the capability to operate both as a GPS receiver and also as a 900 MHz transceiver operating within the ISM band. Since both the GPS and communications functions reside within common radio hardware, they can be linked to provide a positioning capability that leverages both the GPS derived pseudo-range and carrier phase observations and also TOA observations derived from the communications channel.
\end{abstract}

The design of the Positioning and Communication (POSCOMM) Software Defined Radio is described in this paper with the networked architecture used to provide augmented navigation in an urban environment. The POSCOMM network design uses multiple "Master" units, which are operating in locations where they have access to the GPS signals. These can be either ad hoc networked participants or units pre-positioned to provide coverage in areas where GPS has difficulty operating. The Master units transmit a TOA message, which includes a pseudo-random sequence from which the time of arrival at the "Slave" unit can be precisely determined. A message is also sent including the precise time of transmission of the TOA message and the precise location of the Master unit based on the GPS observations. The time-of-arrival differenced with the time-oftransmission provides the Slave unit with a pseudo-range observation from each of the Master unit locations. This can be used to solve for the position of the Slave either using the TOA updates alone or using a combination of both the GPS and TOA observations.

In this paper, simulation and test results are included to demonstrate the accuracy to which the POSCOMM network aided positioning solution can be derived. The POSCOMM combined GPS/TOA solution accuracy is a function of the following components.

- Geometry provided by the Master-Slave relative locations. An analysis is shown of the typical geometry experienced by networked participants in a First Responder application.

- Accuracy of the TOA observations. This is determined by the choice of the communication waveform and the ability to precisely measure the time of reception of the TOA message. Test results are shown presenting the accuracy of measurements when operating inside buildings.

- Accuracy of the GPS time and position mark at the Master unit. Test results are presented showing the ability to precisely position and time sync the SDRs used to broadcast the TOA augmentation signal.

The POSCOMM units are being developed to provide a robust urban navigation solution that can provide precise positioning inside buildings where the GPS signals cannot be received. Applications for this technology include firefighters and other First Responders, also military operations in urban terrain (MOUT).

\section{INTRODUCTION}

A Software Defined Radio (SDR) provides a flexible architecture that allows the same radio components to be reconfigured to perform different functions. NAVSYS has developed an SDR that includes the capability to operate both as a Global Positioning System (GPS) receiver and also as a $900 \mathrm{MHz}$ transceiver operating within the Industrial, Scientific and Medical (ISM) band. Since both the GPS and communications functions reside within common radio hardware, this positioning and communications (POSCOMM) device can use the GPS and communications functionality to provide a positioning capability that leverages both the GPS derived pseudorange and carrier phase observations and also Time-Of-Arrival (TOA) observations derived from the communications channel. The design of the POSCOMM Software Defined Radio is described in this paper.

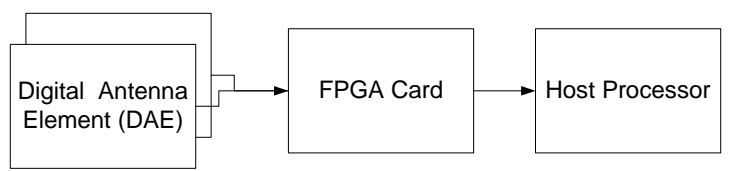

Fig. 1 Software Defined Radio Architecture

The POSCOMM SDRs are designed to operate in a networked architecture, as shown in Fig. 2, where "Master" units are designated as transmitters to provide TOA augmented navigation to "Slave" units operating as receivers in a GPS-denied urban environment. The Master units transmit a TOA message that includes a pseudorandom sequence from which the time of arrival at the Slave unit can be precisely determined. A message is also sent including the precise time of transmission of the TOA message and the precise location of the Master unit based on the GPS observations. The time-of-arrival differenced with the time-oftransmission provides the Slave unit with a pseudorange observation from each of the Master units' locations. This can 
be used to solve for the position of the Slave either using the TOA updates alone or using a combination of both the GPS and TOA observations.

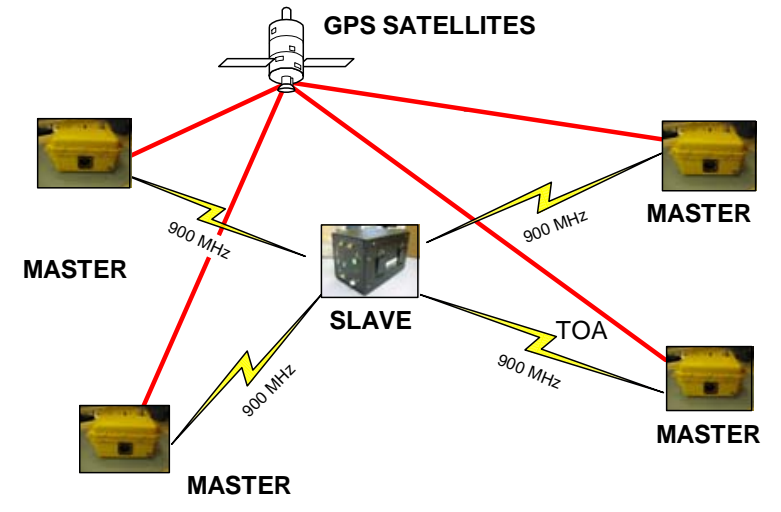

Fig. 2 POSCOMM TOA Network [1]

\section{POSCOMM SOFTWARE DEFINED RADIO}

The POSCOMM GPS/TOA navigation solution was implemented using NAVSYS' Software Defined Radio test bed shown in Fig. 3 [2.]. This has been developed using a modular PC/104 configuration to facilitate rapid prototyping and testing of SDR software applications to support advanced positioning and communications functions. Previously, this SDR has been used for demonstrating a Software GPS Receiver (SGR) Application Programming Interface (API) [3], network assisted GPS operation using the military $\mathrm{P}(\mathrm{Y})$ code GPS signals [4], and also integrated GPS/inertial operation including Ultra-Tightly-Coupled (UTC) GPS/inertial tracking [5].

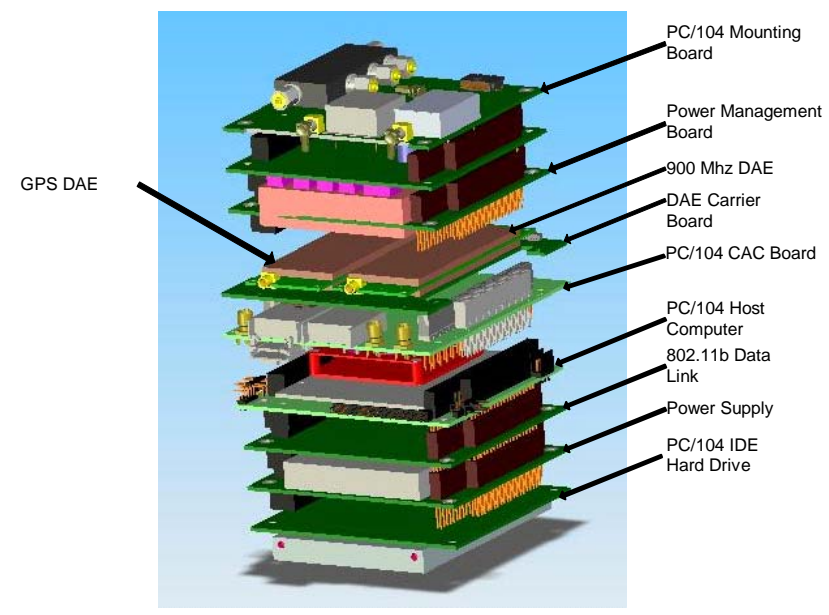

Fig. 3 POSCOMM SDR Components

The POSCOMM SDR system is based on low-cost, commercial-off-the-shelf (COTS) hardware and software. The hardware can use any PC-based environment including desktop, laptop, PC/104, or CompactPCI form factors. Signal processing is performed by a Xilinx Spartan-3 Field Programmable Gate Array (FPGA) card and a Pentium-class CPU. The software is portable and developed for real-time flavors of Windows and Linux operating systems. An SCAbased XML schema is used for system configuration.

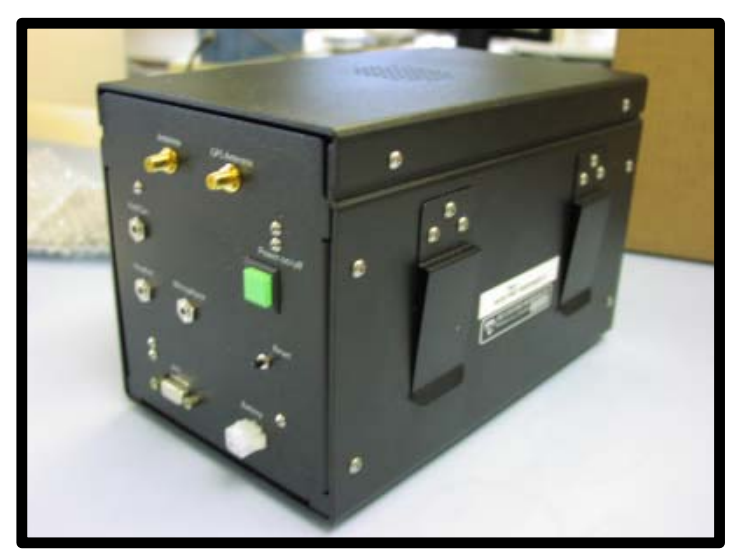

Fig. 4 POSCOMM SDR

Received RF signals for both GPS and TOA are converted to digital signals using Digital Antenna Elements (DAEs). The DAEs have a small 1"x4" size and can be easily modified for alternate frequencies and sampling rates. The DAE is responsible for RF down-conversion and upconversion as well as high-speed A/D and D/A sampling. Each DAE uses a common sample clock and phase-locked reference local oscillator assuring a coherent sampling environment for all transmitted and received signals.

The POSCOMM SDR PC/104 stack shown in Fig. 3 is packaged in the enclosure shown in Fig. 4 and includes the following main components.

- GPS Digital Antenna Element. This is used to receive and track the GPS signals.

- GPS 900 MHz Digital Antenna Element. This includes a $900 \mathrm{MHz}$ receive and transmit channel that is used for either broadcasting or receiving the TOAaided data. This could also be configured for use in communicating between the POSCOMM units. 900 $\mathrm{MHz}$ was selected as this lies in the unlicensed ISM band. The DAE transceiver can be configured though to work at other frequencies.

- 802.11b Data Link. This was used to provide the inter-unit communications link during this phase of testing.

- PC/104 CAC Card. This is a NAVSYS designed card that includes three Spartan FPGAs and a PCI interface to the Host Computer. This interfaces directly with the DAE receive and transmit channels through an adapter board, as shown in Fig. 5.

- Host Computer, Hard Drive and Power Supply. These are COTS components that include a PC/104 form-factor Pentium-M Single Board Computer, power supply and 80 GByte Hard Drive 


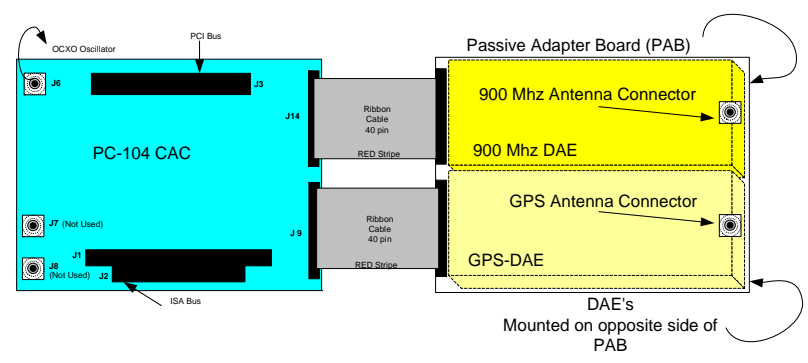

Fig. 5 PC/104 CAC to DAE Interfaces

\section{POSCOMM SDR OPERATION}

The POSCOMM SDRs are configured through software to operate as either a Master (Transmit) or Slave (Receiver) mode of TOA operation.

Master units are required to be tracking at least one GPS satellite to allow the time of the TOA transmission to be synchronized precisely with GPS time. These units send TOA Assistance messages across the network which tells the Slave units what TOA observations are available for use in aided navigation and also provide the location of the Master units that are providing the TOA aiding.

Slave units will default to GPS tracking if satellites are in view, but are not required to track any GPS satellites for them to operate. At start-up, they initialize time across the network using Network Time Protocol (NTP). On receipt of the TOA Assistance messages from the Master units, the Slave unit will then initiate tracking of the TOA observations which will be used, in combination with any observed GPS satellites, to compute the aided navigation solution.

The GPS/TOA solution accuracy is a function of the following components which are addressed in the POSCOMM SDR design and described in the following sections of this paper.

- Accuracy of the GPS time and position mark at the Master unit.

- $\quad$ Geometry provided by the TOA observations.

- Accuracy of the TOA observations

TABLE I

TOA Acknowledge Message

\begin{tabular}{|lll|}
\hline \multicolumn{1}{|c|}{$\begin{array}{c}\text { Field } \\
\text { Name }\end{array}$} & Units & \multicolumn{1}{c|}{ Description } \\
Time & Week & $\begin{array}{l}\text { GPS time of week of first TOA being } \\
\text { transmitted }\end{array}$ \\
secs & & $\begin{array}{l}\text { ID of PRN code } \\
\text { Interval between TOA signals }\end{array}$ \\
Period & ms & $\begin{array}{l}\text { Duration of TOA ranging signal } \\
\text { Duration }\end{array}$ \\
Freq & $\mathrm{ms}$ & RF Frequency of TOA signal \\
\hline
\end{tabular}

TOA TRANSMISSIONS

The SDR architecture allows for a variety of different waveforms to be used to provide TOA assistance. The key feature of the POSCOMM SDR approach is that the design of the SDR DAEs and firmware allows the timing of the TOA transmission to be precisely locked in time to the received
GPS signals. For the POSCOMM testing, we implemented a combined Code-Division Multiple Access (CDMA), TimeDivision Multiple Access (TDMA), and Frequency-Division (FDMA) approach for sharing the spectrum between the multiple Master Units providing TOA assistance. This provides maximum flexibility in configuring the POSCOMM TOA assistance network to optimize performance and share limited bandwidth for both positioning and communications functionality. The CDMA, TDMA and FDMA parameters that specify the TOA signal characteristics are all defined using configuration parameters and are defined in the TOA ACK Message sent by the Master Units (see TABLE I).

\section{GEOMETRY OF TOA-AIDED SOLUTION}

The horizontal and vertical accuracy of a GPS TOA-aided solution is a function of the TOA dilution of precision (DOP) scaled by the TOA observation accuracy. The worst-case performance occurs when no GPS satellites can be tracked and only TOA observations are available for navigation.

If only ground-based TOA transmitters are used, then the 3-D TOA solution is indeterminate and the DOP approaches infinity (Fig. 6). If altitude-aiding is used, for example from a baro-altimeter, then a 3-D solution can be calculated. Fig. 7 shows the simulated dilution of precision with 4 transmitters located around a 3-story building where $\mathrm{HDOP}<1$ and $\mathrm{VDOP}<1$.

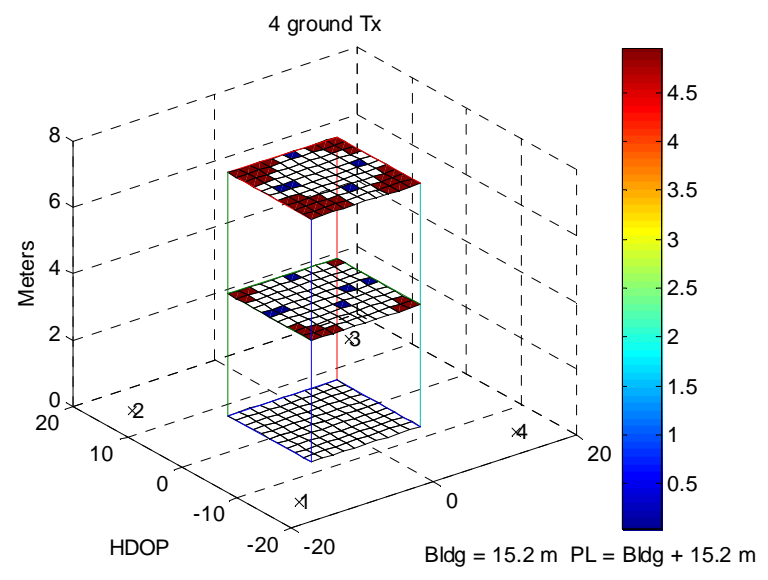

Fig. 6 HDOP with 4 Ground-Based Transmitters 


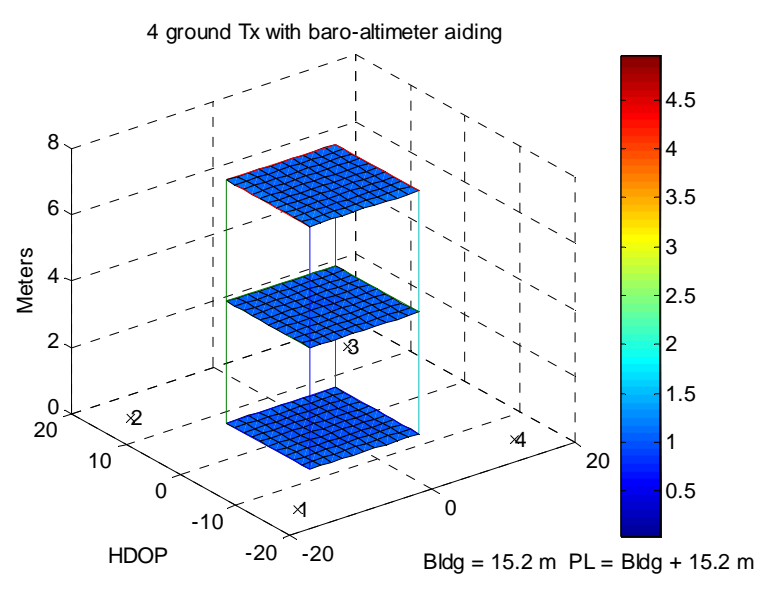

Fig. 7 HDOP with 4 Ground-based Transmitters and Altitude-aiding

\section{TOA OBSERVATIONS}

The accuracy of the TOA observations is a function of the waveform characteristics, the tracking loops employed and the environment. The main challenge faced for the TOA ranging signal design is to provide robust and accurate performance in the presence of multipath.

To evaluate the multipath environment and the ability of the TOA tracking loops to handle these errors, four Master units were set up around the NAVSYS building shown in Fig. 8 with the test layout shown in Fig. 9. Test results were collected from units operating both outside the building, where GPS could be used as a truth reference, and inside the building. In both cases, the TOA signals were passing through multiple different types of construction. The west end of the building is metal construction while the center and east end is brick construction.

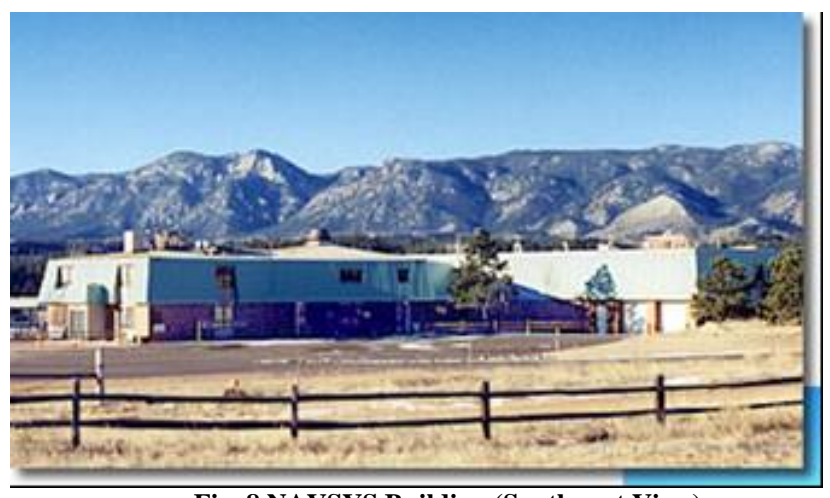

Fig. 8 NAVSYS Building (Southwest View)

A maximum likelihood estimation (MLE) algorithm is used to estimate the TOA from the correlation results generated from the $900 \mathrm{MHz}$ received signal correlated with the modulated PRN code. The algorithm detects the peak of the correlation from the closest in signal detected. This will result in detecting the correlation peak of the signal from the direct path from the transmitter rather than a multipath signal that arrived from an indirect path. Fig. 10 shows the correlation results from four transmitters when the receiver has a direct line-of-sight to the transmitters. All four signals have a strong detected correlation peak with a received RF signal of around $-16 \mathrm{dBm}$. Fig. 11 and Fig. 12 show the correlation results from signals received through the NAVSYS building. In these cases, the building can significantly attenuate the received signal power and also strong multipath signals are present, which appear as peaks showing to the left of the direct signal peak. The MLE algorithm used to perform the TOA tracking detects the closest peak in each case shown.

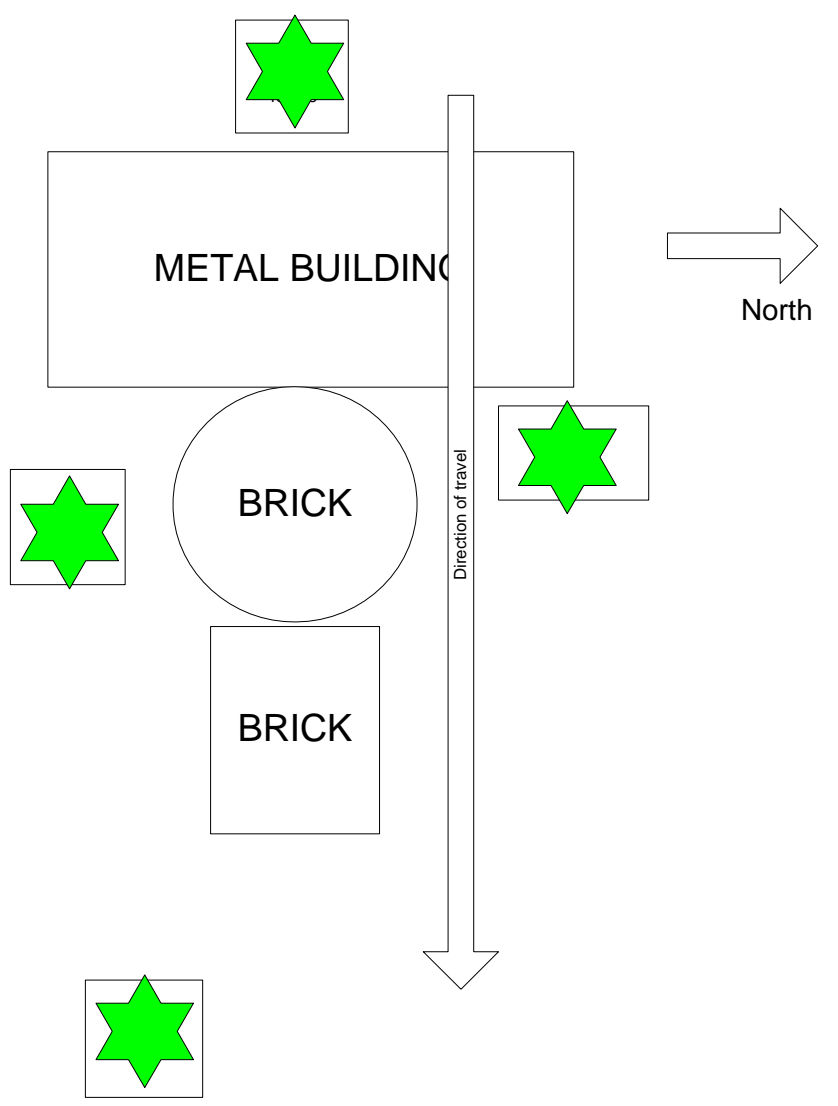

Fig. 9 Indoor Test Pseudolite Layout
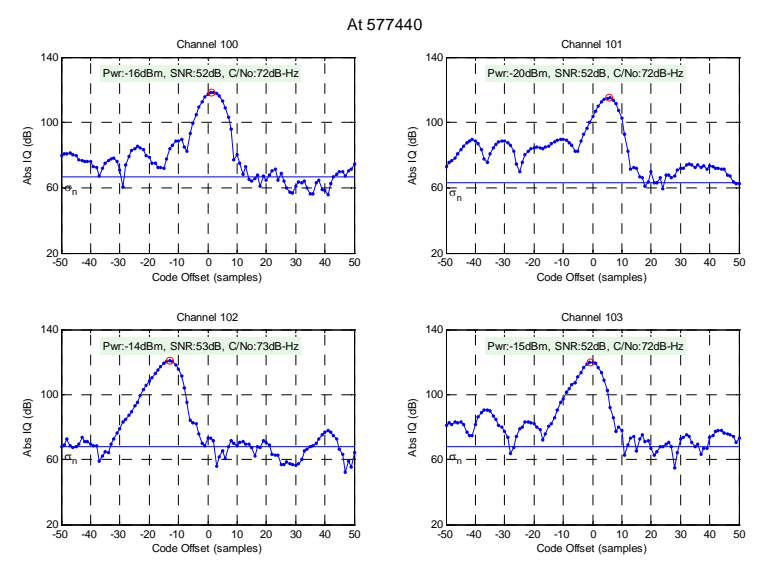

Fig. 10 MLE Estimation of Shortest TOA Pseudorange (outdoor testing) 


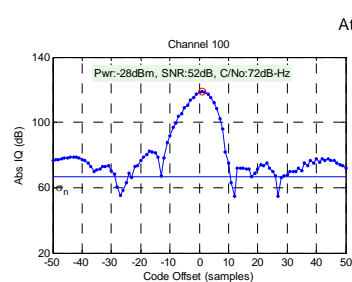

At 590850
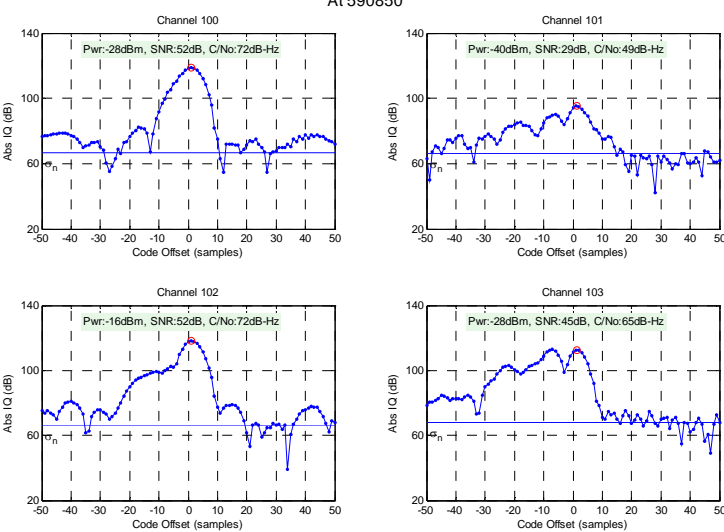

Channel 103

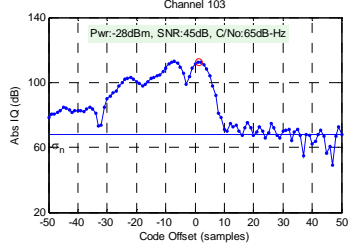

Fig. 11 MLE Estimation of Shortest TOA Pseudorange (indoor testing)

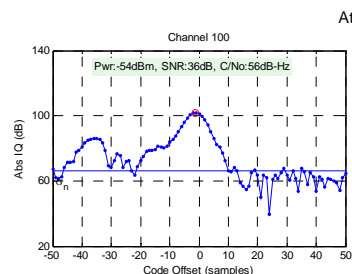
At 590340
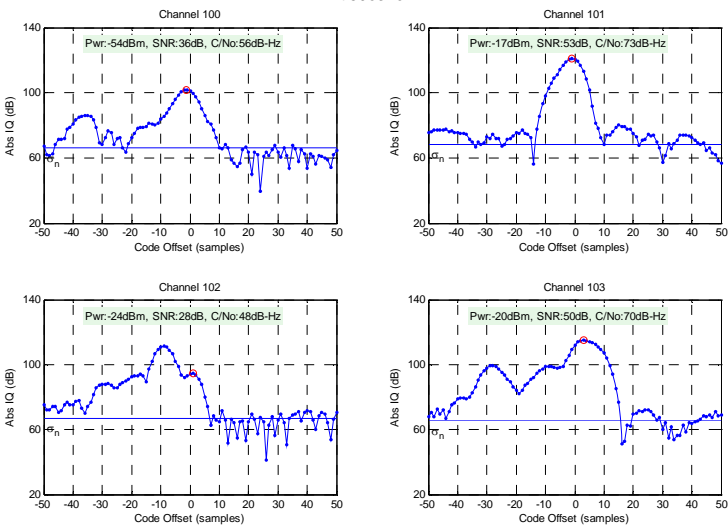

Channel 103

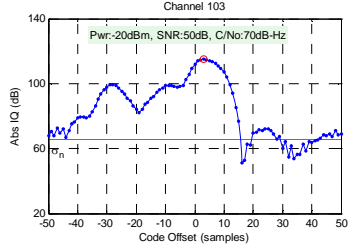

Fig. 12 MLE Estimation of Shortest TOA Pseudorange (indoor testing)

\section{TOA-AIDED NAVIGATION TEST DATA}

The TOA-aided navigation solution was first tests in an outdoor environment using GPS as truth data to analyze the performance of a TOA navigation solution. The results are shown in Fig. 13. The POSCOMM navigation solution computed from four TOA observations agreed with the GPS truth solution to within 5 meters except for a few excursions.

In Fig. 14 the indoor test configuration is shown that was used to test the TOA solution. Five transmitters were set up at different sites around NAVSYS's building. This building uses brick construction on the east side and center and is a metal building with brick façade on the west side. The navigation test results are shown in Fig. 17. The average position error was on the order was $5.53 \mathrm{~m}$ (RMS)

An example of the signal variation throughout the building is shown in Fig. 15 and Fig. 16. This data is shown for a Master transmitter located on the SE side of the building. The figures show that the received SNR of the TOA signals drops by $40 \mathrm{~dB}$ as the unit is moved to the West of the building. These plots also compare the tracking performance achieved with the straight peak detection and the MLE estimation. The MLE estimation technique noticeably has fewer excursions demonstrating its improved performance in tracking the TOA signals in a strong multipath environment.
Further improvements are being added to the design of the Master and Slave POSCOMM SDRs to improve on the accuracy of these initial results. In the next phase of the project, we will also be integrating a MEMS IMU with the POSCOMM SDR to assist in filtering and tracking the TOA observations and for use in an integrated TOA/inertial navigation mode.

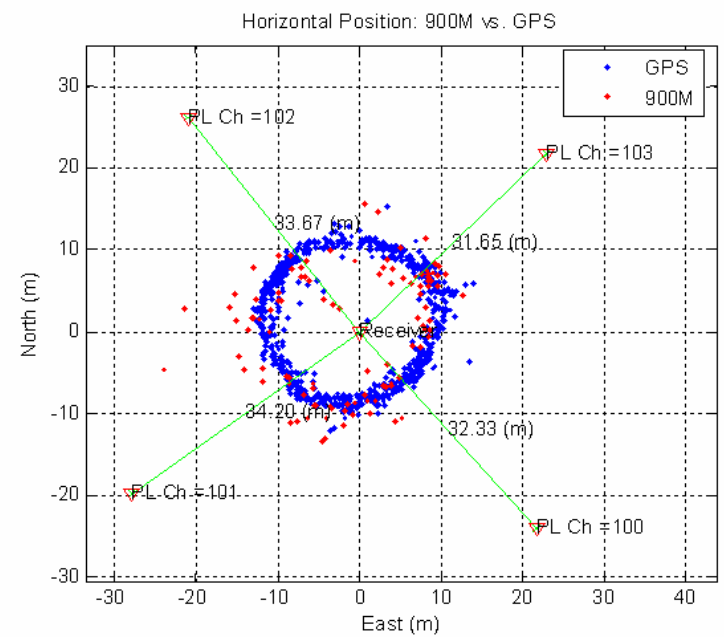

Fig. 13 TOA Navigation Solution

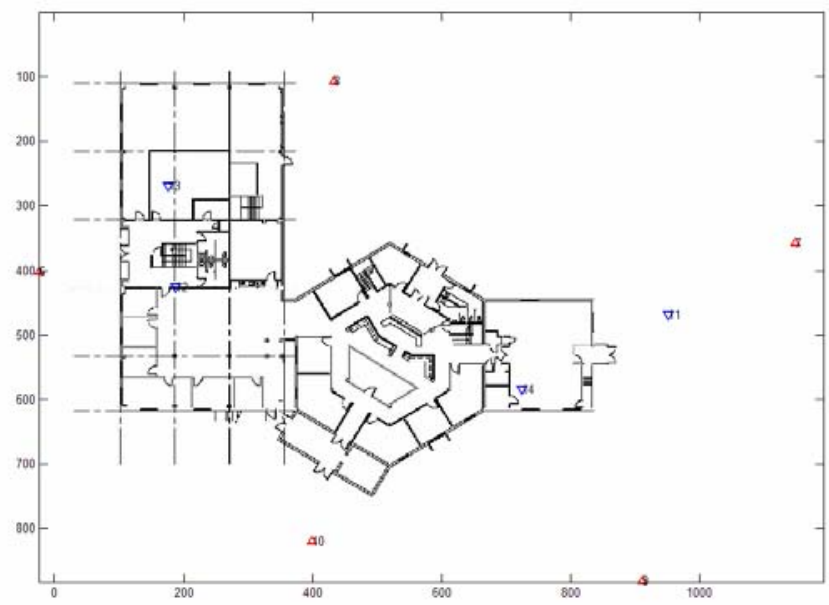

Fig. 14 NAVSYS Building Transmitter Sites (Red) and Test Sites (Blue) 


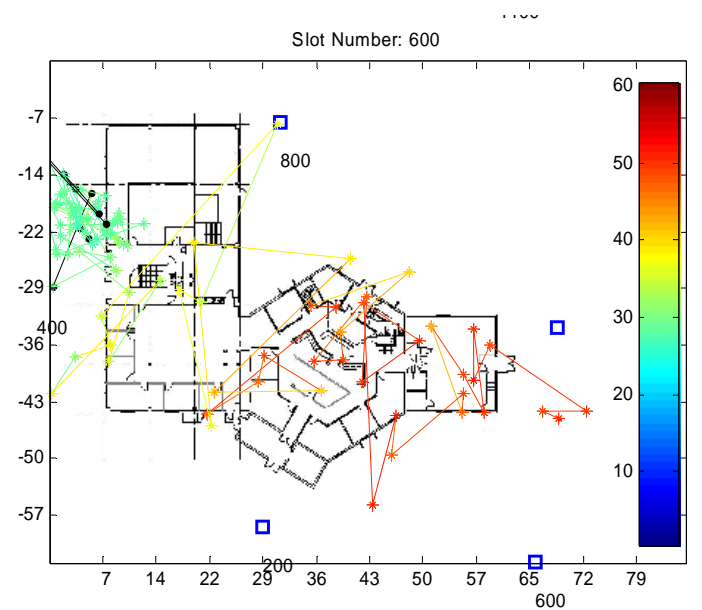

Fig. 15 Signal Strength using TOA Peak Detection

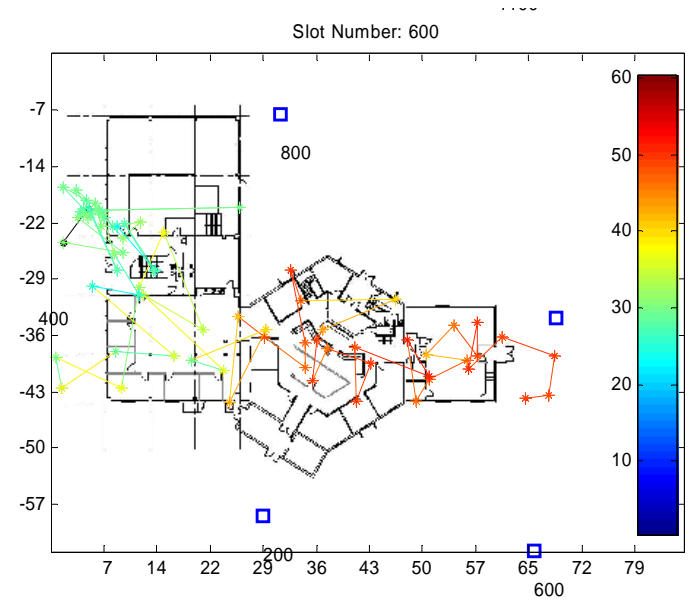

Fig. 16 Signal Strength using MLE Detection

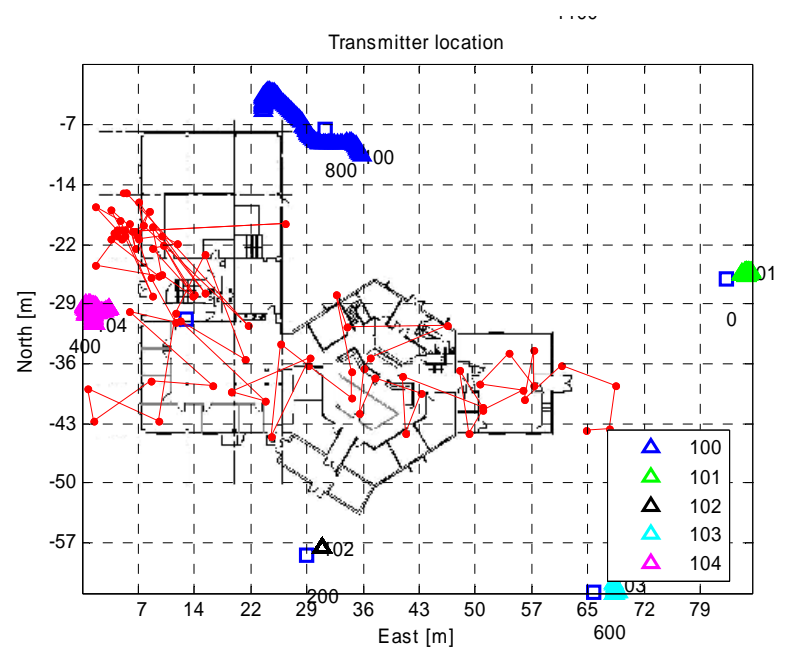

Fig. 17 Navigation solution in mobile indoor test

\section{CONCLUSION}

The POSCOMM units are being developed to provide a robust urban navigation solution that can provide precise positioning inside buildings where the GPS signals cannot be received. Military applications for this technology include improved military operations in urban terrain (MOUT). Commercial applications include firefighters as well as other first responders. This project will give firefighters, police officers and emergency officials an electronic vest and eyepieces that will provide their commanders with their location and their vital signs, as well as real-time video of their surroundings.

The initial tracking and positioning results shown in this paper show the capability provided by the POSCOMM SDR to augment GPS signal tracking in the challenging urban environment with TOA aiding from an alternative RF source. This technology offers the capability to provide access to GPS-like quality of service both outside and inside buildings.

\section{ACKNOWLEDGMENTS}

The authors would like to acknowledge the support of CERDEC, STTC, and Rex Systems Incorporated who have provided funding to support the development of this technology.

\section{REFERENCES}

[1] Systems shown from Rex Systems Incorporated First Responder Program

[2] A. Brown, Y. Lu, and J. Nordlie, "Integrated GPS/TOA Navigation using a Positioning and Communication Software Defined Radio,” Proceedings of ION GNSS 2005, Long Beach, California, September 2005.

$\left[{ }^{3}\right]$ F. Carpenter, S. Srikanteswara, and A. Brown, "Software Defined Radio Test Bed For Integrated Communications And Navigation Applications,” Proceedings of 2004 Software Defined Radio Technical Conference, Phoenix, Arizona Nov. 2004

$\left[{ }^{4}\right]$ A. Brown and P. Olson, "Urban/Indoor Navigation using Network Assisted GPS”, Proceedings of ION $61^{\text {st }}$ Annual Meeting, Cambridge, Massachusetts, June 2005

$\left.{ }^{5}\right]$ A. Brown and Y. Lu, "Performance Test Results of an Integrated GPS/MEMS Inertial Navigation Package,” Proceedings of ION GNSS 2004, Long Beach, California, September. 2004 